\title{
A Look at Compressor Impeller Technologies for Turbochargers Focusing on Surge Mitigation
}

\section{Rachel Schwind ${ }^{1 *}$ and Shaaban Abdallah ${ }^{2}$}

${ }^{1}$ Department of Mechanical and Materials Engineering, University of Cincinnati, Cincinnati, Ohio, USA

${ }^{2}$ Department of Aerospace Engineering and Engineering Mechanics, University of Cincinnati, Cincinnati, Ohio, USA

\begin{abstract}
With rising fuel costs and new emissions standards for automobiles, automotive manufacturers are turning to new ways to decrease engine size and emissions while still maintaining high power operation. This can be accomplished through the use of turbochargers. The aim of this study is to analyze what is currently being done with turbocharger compressor technology to meet these requirements. This analysis will focus on different impeller blading designs including splitter bladed impellers, tandem bladed impellers, and tandem bladed impellers with a casing blade. There are different advantages associated with each impeller design. The tandem bladed impeller designs are shown to have decreased efficiency and pressure ratios as compared with the backswept and/or splitter bladed designs. It is also shown that the operating range is increased with the tandem design due to a lower surge margin. Further analysis needs to be conducted on refining the tandem bladed and tandem bladed with a casing blade designs to truly see if these designs have the potential for greater performance improvements.
\end{abstract}

Keywords: Centrifugal compressor impeller; Turbocharging; Turbomachinery; Tandem-blading; Compressor surge; Compressor performance; Impeller designs

\section{Introduction}

With rising fuel costs and the depletion of natural resources, automotive manufacturers have been looking for ways to increase fuel economy without significantly decreasing an engine's power output. Many engine manufactures have turned to the use of turbochargers to meet this need. A turbocharger is able to increase an engine's power by forcing more air into the combustion chamber thus also allowing it take in more fuel as well. The mass of air entering the combustion chamber can increase the power output as well as allow for leaner combustion. These are an attractive option for modern cars because they allow manufacturers to use smaller engines for the same output power. This can increase fuel economy at idling stages as well as reduce the overall weight of the vehicle. They also function as a way to reduce emissions since a majority of modern turbochargers are driven by an exhaust gas turbine. The turbine is connected to the compressor via a common shaft so when it pulls in the exhaust gases, it generates the rotation necessary for the compressor to operate.

A major component of the turbocharger is the compressor which is generally a centrifugal compressor. Flowgenerally enters the compressor in axial direction. The rotating impeller blades then accelerate the fluid before discharging it radially. This increase in velocity will generally lead to an increase in pressure as well. The accelerated fluid can then be discharged into a collector and then on to a diffuser where the fluid velocity is decreased allowing further conversion of velocity into pressure. Generally, a centrifugal compressor is designed for optimal operation at a single speed. This can be problematic as compressors need to be able to function at off-design speeds, especially in the case of automotive turbochargers which have highly transient operation.

Due to the transient nature of engine operation, a turbocharger ideally has a large operating range. Bounding the operating range at low speeds with high pressure ratios is the surge limit. Surge occurs when there is a low flow rate at a relatively high pressure ratio causing flow reversal. This causes fluctuations in the flow resulting in unstable operation. Surge that results in a flow reversal over almost the entire fluid flow, also known as deep surge, can lead to catastrophic failure. Light surge with smaller flow reversal areas will not necessarily cause a failure of the system and the compressor may still operate, but the performance will suffer. While it may not be a completely catastrophic failure, it could result in engine misfires if not enough air is fed into combustion chamber. Any operation in the surge margin can be harmful to the performance of both the turbocharger and the engine and should be avoided at all costs. Bounding the operating range at high speeds and mass flow rates is the choke limit. Generally the choke limit occurs when the flow velocity reaches a maximum of sonic speed. This limit is largely determined by the throat area at the inlet.

\section{Current Impeller Designs}

As previously mentioned, surge is a major limiting factor in the operating range of a centrifugal compressor. The necessity for a large operating range to prevent turbocharger surge and stalling has led manufacturers to look at ways to mitigate surge effects and increase the overall operating range of the compressor. While using multiple compressors in a row can lead to a large overall pressure ratio by successively increasing the ratio through each stage while keeping the ratio of the single stage relatively low, this method is not ideal for use in a turbocharger application as it increases the overall size of the entire compressor unit. The new goal for turbocharger manufactures is to develop high pressure ratio centrifugal compressors comprising of a single impeller. This will ideally decrease the overall size of the compressor while still providing the necessary pressure ratio change.

*Corresponding author: Rachel Schwind, Student Department of Mechanical and Materials Engineering, University of Cincinnati, Cincinnati, Ohio, USA, E-mail: schwinra@mail.uc.edu

Received July 02, 2015; Accepted July 06, 2015; Published July 16, 2015

Citation: Schwind R, Abdallah S (2015) A Look at Compressor Impeller Technologies for Turbochargers Focusing on Surge Mitigation. Global J Technol Optim 6: 185. doi:10.4172/2229-8711.1000185

Copyright: (c) 2015 Schwind R, et al. This is an open-access article distributed under the terms of the Creative Commons Attribution License, which permits unrestricted use, distribution, and reproduction in any medium, provided the original author and source are credited. 
Due to the demand for improving centrifugal compressor performance, researchers have been investigating many different ways to increase operating ranges and off-design performance. Some compressors employ active methods of surge control such as adjustable vanes and controls [1], but the cost of these methods makes them impractical for use with mass produced turbochargers. Passive control methods such as a recirculating bleed port are also being studied for implementation on centrifugal compressors. For the purposes of this state of the art analysis, focus is being placed upon the performance impact of different impeller blade configurations. Further analysis may prove that incorporating an optimized blade configuration with a passive surge control method may produce better performance than the designs employed separately. That analysis is not within the scope of this study, but could be a potential area for future work.

\section{Impeller with backswept blades}

Many standard impellers are designed with simple radial blades due to their ease of manufacture. However, as the demand for better efficiencies and increased operating ranges began to grow, designers began to look at utilizing backswept blades because they offered a higher surge margin as compared with radial or front-swept blades [2]. Figure 1 shows an unshrouded backswept impeller.The backswept impeller has a varying blade angle from the tip of the blade to the hub which sweeps back in the direction opposite of the rotation [3]. While these are generally more favored than a standard radial impeller, Wang et al. found that the backswept blades have a reduced pressure ratio compared to that of a standard radial impeller [4]. This study also shows that the isentropic efficiency and volume flow rates are increased at lower velocities for the backswept design. Even though the pressure ratio may not be as high, the performance improvements at lower speeds are beneficial due to the potential to increase of the viable operating range of the compressor.

\section{Impeller with splitter blades}

One approach looking to increase the operating range and efficiency of the impeller was the addition of splitter blades. A splitter blade is a blade that does not extend the full length of the impeller and is generally placed between two full length blades. The number of full length blades on an impeller is limited by the dimensions of the hub at the inlet.The circumferential distance between the blades increases as the blades extend from the inlet to the outlet. This provides more space for the flow to expand and potentially reach stagnation causing a flow blockage. The addition of splitter blades can provide additional guidance to the flow towards the exit while still keeping the number of blades at the inlet low (Figure 2).

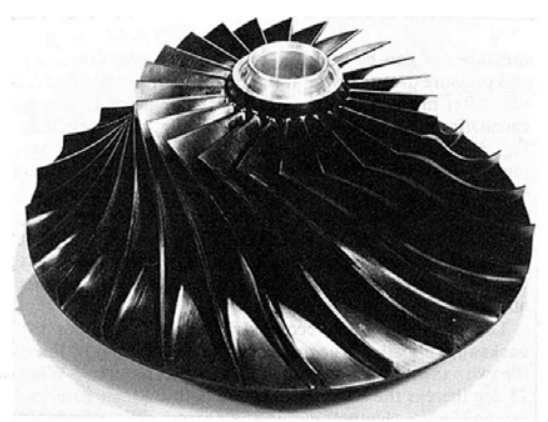

Figure 1: Example of a $30^{\circ}$ backswept impeller designed and tested by Krain [3].

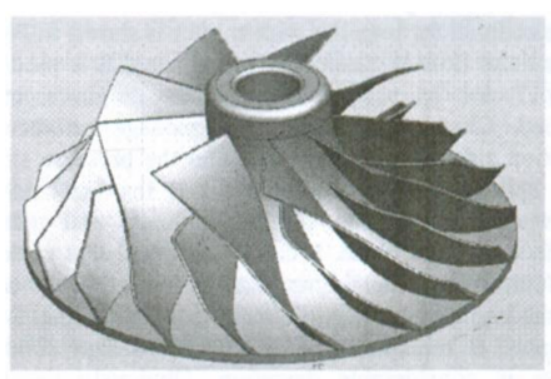

Figure 2: Impeller with splitter blades [5].

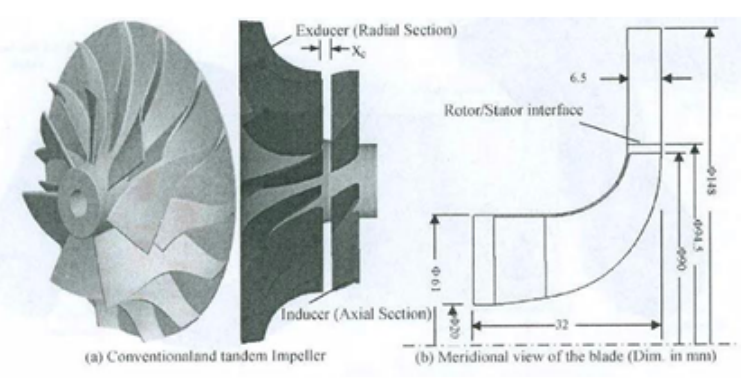

Figure 3: Conventional and tandem bladed impeller geometries [6].

Nassar et al. conducted a parametric study on splitter blades since most centrifugal compressors have adopted this design. Other than the added benefit of extra guidance through the radial portion of the impeller, splitter impellers are also employed because a greater number of blades are needed to prevent stagnation in the radial part of the flow path due to the Coriolis Effect on the blade-to-blade pressure gradient. These functions allow for an improvement in mass flow rate and improvement of efficiency at off design conditions [5].

\section{Tandem bladed impeller}

A tandem bladed impeller takes the typical impeller design and modifies it such that there is a cut which splits the blade axially into two separate blades, one acting as the inducer and one acting as the exducer. The inducer and exducer blades can be directly aligned such that they still follow a similar shape to that of a typical centrifugal impeller blade or they can be circumferentially offset from one another. Figure 3 shows the geometry of a tandem bladed impeller without clocking as compared to a conventional impeller. While most constructions have the split between the inducer and exducer portions of the blading, there are also constructions that have the blade split further down the blade, into the radial section.

As noted by Danish et al., very few publications are available which study tandem bladed configurations for radial turbomachinery [6]. There has been extensive research into their application in axial turbomachines which have shown improvements over conventional design [7]. For this reason attention has turned towards making this a viable option for centrifugal applications.

Danish et al. compared a tandem bladed turbocharger compressor stage with that of a standard backswept impeller with splitter blades [6]. They concluded that the overall efficiency of the impeller is lower than that of the conventional design used for the comparison. However, the tandem bladed design did provide better results near choke flow and the surge point appears to be lower for the tandem design. Ju and 
Zhang looked at the design optimization for a tandem bladed impeller and then compared the performance of the optimized design with that of a standard impeller with splitter blades [7]. It was determined that the tandem impeller had lower efficiency and pressure ratio than the conventional impeller, but it achieves improved flow qualities at the impeller exit. Danish et al. conducted a performance evaluation of a tandem bladed centrifugal compressor against a conventional centrifugal compressor [8]. It was once again found that while the impeller efficiency and the pressure ratios were lower, the surge margin was increased and the tandem design showed better performance near choke flow. Design optimization for a tandem bladed impeller has been started by Hlavacek [9]. At this stage in the design development, it has been determined that there is a real possibility for the improving the stage characteristics.

\section{Tandem bladed impeller design with casing blade}

A design which employs two rows of blades on the impeller and one row on the stationary casing was patented by Dr. Shaaban Abdallah. The blades are spaced such that the casing blade passes through the radial gap between the two impeller blades as the impeller rotates [10]. Figure 4 shows this design concept.

This design allows for each blade row to be refined separately to potentially provide a better overall performance. While the tandem bladed design could also allow for this flexibility, the additional blade row on the stationary casing helps redirect the flow before it goes into the second impeller blade row. This then provides additional flexibility in the orientation of the second impeller blade row because it does not need to be related to the orientation of the velocity of the fluid coming off of the first blade row. Currently, research regarding the performance of this design is being conducted through computational methods at the University of Cincinnati. An initial study was completed by Ellsworth and Turner which indicated that this multistage design was able to achieve higher pressure ratio, but had a lower efficiency when compared to the backswept design of Krain [11].

\section{Discussion}

The use of backswept impeller blades and splitter blades has been common in centrifugal compressors for years which is why most new designs are compared to these designs or to the standard radial impeller. The backswept impeller blade does appear to increase the operating range over the standard radial impeller, but as noted previously, the number of blades needed to prevent stagnation increases in the radial

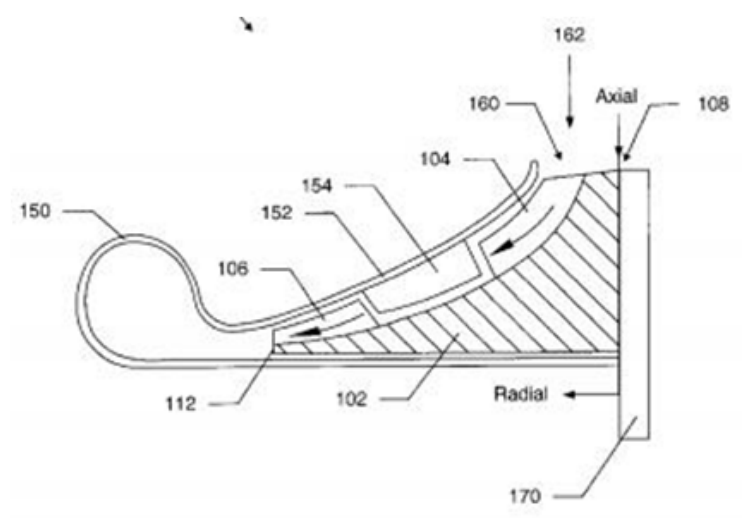

Figure 4: Cross-sectional image illustrating one embodiment of the patented design [10]. portion of the impeller as compared with the axial inlet. While one option would be to increase the number of blades everywhere, this could actually lead to more problems due to a reduction in the throat area at the inlet. This could then lead to insufficient air flow through the compressor and thus less efficient operation or even possible choking. For this reason splitter blades are employed so that the additional blading necessary for the radial portion can be implemented without causing blockages near the inlet. This allows for an increase in the mass flow and off design efficiency as compared with the backswept impeller.

Since the maximum ability of an impeller blade to accelerate a fluid is related to the length of the blade, the impeller with splitter blades seems to provide a good solution by allowing the longer blades with additional blading in the radial section. The smaller blades associated with either of the tandem type designs may not be able to accelerate a fluid as much as a conventional blade which extends along the length of the impeller. However, the additional blade refinement afforded by having blades which are discontinuous along the hub as well as the potential to have an increased number of discrete blades over conventional designs may allow for significant increases in fluid flow acceleration by these designs. The longer blade length associated with the full bladed designs can also lead to boundary layer separation as the flow continues along the single blade. The tandem bladed configuration allows for the reformation of the boundary layer on the second cascade of rotor blades. This reformation helps increase the flow uniformity at the impeller exit which could then lead to an increase in efficiency of the overall compressor stage. A non-uniform discharge can actually negatively impact the overall stage efficiency [8]. This indicates that the overall stage efficiency could actually be increased even if the impeller efficiency decreases. As mentioned previously, turbocharger compressors require a large operating range for optimal functionality. For this reason, the reduction in impeller efficiency may not be as large of a concern, if the desired operating range can be achieved. While the tandem designs may have reduced performance in the optimal operating range, the potential improvements to performance in the off-design conditions may be more valuable. For this reason the tandem bladed impellers may actually be more suited for turbocharger application.

It should be noted that extensive design optimization beyond looking at clocking fraction and clearances has not been conducted on the tandem designs so there may be additional room for improvement that has not yet been realized. Currently, the analysis conducted on tandem bladed impellers has looked at the optimized configuration as it relates to maintaining a similar blade curvature to the baseline impeller. While this is good for comparison between the two design types, there is the opportunity for further refinement because the limitations associated with a single blade configuration have been removed. The tandem bladed impeller with a casing blade design provides the most flexibility in this area as the design for each of the blade rows on the rotor is not dependent upon the flow conditions on the other blade row due to the presence of the stator blade. While a full analysis of the performance of an impeller with this design has not been completed yet, the potential can be seen based upon the performance results from the standard tandem bladed impeller and the notion that further refinement is possible.

Another potential advantage of the tandem type designs is that the second blade row is not constrained to the first blade row so the number of blades can be refined for each location. This can play a significant role in the development of this technology as compared to those with splitter blades as the losses due to friction will increase 
Citation: Schwind R, Abdallah S (2015) A Look at Compressor Impeller Technologies for Turbochargers Focusing on Surge Mitigation. Global J Technol Optim 6: 185. doi:10.4172/2229-8711.1000185

with the number of blades due to the increase in surface contact area. By being able to refine each blade row independently, the number of blades may be able to be reduced as there would be more flexibility in their circumferential location. As previously noted, further research needs to be done into the overall refinement of both the tandem bladed impeller and the tandem bladed impeller with a casing blade. Outside of turbocharger applications, the tandem bladed impeller designs are also being investigated for use as a separator in two phase flow applications. As noted by Matsushita et al. this could reduce the necessity of needing both a compressor and a pump in two phase flow applications [12].

\section{Conclusions}

It is important to analyze the full requirements of the system of interest before choosing the correct impeller design. In applications with limited space such as a turbocharger, a single stage centrifugal compressor is ideal. As mentioned this can limit the overall operating range for the compressor, potentially leading to poor performance since the turbocharger is a highly transient application. It is noted that while the tandem impeller design shows an improvement in operating range, it also shows a decrease in overall efficiency. For the turbocharger application, a large operating range is extremely desirable so it may be worth sacrificing some efficiency to achieve. This will be largely dependent upon the application's needs.

As noted in the previous sections, there has not been a lot of analysis done on tandem bladed impellers recently so further research into this design is recommended. In the research found during this study, there did not appear to be any studies looking at refining the two blades independently other than looking at clocking fraction. Future work could be focused on looking at different blade geometries for the top blade section and bottom blade section to see if more efficient performance can be obtained while still maintaining the increased operating range. Since the top blade row leads flow directly into the second blade row, careful attention should be paid to how the blades are being adjusted in relation to one another.

Further analysis also needs to be conducted on the tandem bladed impeller with a casing blade. The current literature search was unable to find any specific analysis associated with this design. However, based upon the similarities to other designs, this impeller design should provide an increase in the operating range over the standard centrifugal impeller as well as an increase in outlet flow uniformity. Further studies should be conducted to optimize the design and compare it to both the baseline impeller and the tandem bladed impeller. This analysis would prove very useful in future turbocharger designs as it may help manufacturers decided what impeller type will best suit their needs.

It may also prove worthwhile to perform a cost analysis study on newer designs. The manufacturing of standard impellers is already complex and an area of concern for some manufacturers. With advancements in impeller designs, the costs of machining and the precision necessary are both rising. Being able to illustrate performance enhancement versus the overall cost of the more complex geometry may help manufacturers decide on the best option for their application. Additionally, in order for researchers to convince companies to utilize these new impellers, it will be necessary to show that the additional manufacturing cost will be worth it due to performance improvements.

\section{References}

1. Schleer MW (2006) Flow structure and stability of a turbocharger compressor. Ph.D. Dissertation, Federal Institute of Zurich, Switzerland.

2. Rangwala AS (2005) Industrial gas and steam turbines, Turbo-machinery dynamics: Design and operation, (1stedn). McGraw-Hill, New York, 89-94

3. Krain H (1987) Swirling Impeller Flow.J Turbomach 110(1): 122-128.

4. Wang T, Peng C, Wu J (2014) Back swept angle performance analysis of centrifugal compressor. Mechanika 20(4): 402-406.

5. Nassar A, Hagpurwala QH, Reddy KB (2006) Design and CFD investigation of centrifugal compressor for turbocharger and parametric study on splitter blades.Sas Tech 2: 35-41.

6. Danish SN, Qureshi SR, EL-Leathy A, Khan SU, Umer U, et al. (2014) Numerical investigation \& comparison of a tandem-bladed turbocharger centrifugal compressor stage with conventional design. Journal of Therma Science 23: 523-534.

7. Ju YP, Zhang CH (2014) Design and optimization and experimental study of tandem impeller for centrifugal compressor. Journal of Propulsion and Power 30: 1490-1501.

8. Danish SN, Kahn SU, Umer U, Qureshi SR, Caochen M (2014) Performance evaluation of tandem bladed centrifugal compressor. Engineering applications of computational fluid mechanics 8: 382-394.

9. Hlavacek D (2014) Recent progress in developing a tandem-bladed centrifugal compressor stage. STC Conference.

10. Abdallah SA (2001) Patent No. 6589013. United States of America.

11. Ellsworth G, Turner M (2013) Stages centrifugal compressor: Final Report Cincinnati: University of Cincinnati.

12. Matsushita N, Furukawa A, Okuma K, Watanabe S (2005) Influences of impeller diameter and diffuser blades on air-water two-phase flow performance of centrifugal pump. Proceedings of the ASMEFluids Engineering Division Summer Meeting and Exhibition. 